\title{
Highly efficient deep desulfurization of fuels by chemical oxidation
}

M. C. Capel-Sanchez, P. Perez-Presas, J. M. Campos-Martin*, J. L. G. Fierro

Instituto de Catálisis y Petroleoquímica, CSIC. Marie Curie, 2 Cantoblanco, 28049 Madrid, Spain, http://www.icp.csic.es/eac/

* Corresponding author

E-mail: j.m.campos@icp.csic.es

Fax: +34 915854760 


\begin{abstract}
This paper describes the oxidation of several model S-containing molecules with hydrogen peroxide in $\mathrm{L}-\mathrm{L}$ phase system using a heterogeneous catalyst under atmospheric pressure in the 333-353 $\mathrm{K}$ temperature range. Molybdenum and tungsten compounds are prepared by anion exchange with alkylammonium derivatives covalently anchored to silica gel. These solids are robust heterogeneous catalysts able to activate selectively hydrogen peroxide to remove sulfur compounds via oxidation (ODS). The influence of several reaction variables (the support, the reaction temperature, the nature of the substrate, the solvent, the molar ratio of the oxidant $\left(\mathrm{H}_{2} \mathrm{O}_{2}\right)$, the S-containing molecule, the catalysts nature and the reuse of the catalysts) on the performance was examined. The potential of this methodology is illustrated by the complete S-removal from a 0.2 wt\% dibenzothiophene mixture at $353 \mathrm{~K}$ in less than $1 \mathrm{~h}$ of reaction. Molybdenum catalysts exposed to hydrogen peroxide form peroxomolybdates moieties which are more active than acid precursors. The activated Mo catalysts are very active in ODS reaction and can be reused four times without lose of activity.
\end{abstract}

Keywords: Liquid phase reactions; ODS; Oxidation; Hydrogen peroxide 


\section{INTRODUCTION}

The oil industry is facing increasing pressure to remove organic sulfur compounds from transportation fuels. Each refinery must meet unique circumstances, such as diesel blend components and hydrogen availability.

Current hydrodesulfurization technology (HDS) allows desulfurize aliphatic and acyclic sulfurcontaining compounds quite efficiently when adopted at industrial scale. This process, however, is limited when treating dibenzothiophene (DBT), especially in the case of DBTs having alkyl substituents at position 4 and/or 6 [1]. Thus, the production of ultra-clean light oil inevitably requires severe operation conditions and especially highly active catalysts. In the development of any alternative energy-efficient desulfurization process a radical approach, not limited to conventional HDS technology, is required. To remove undesirable sulfur-containing compounds or to convert them into more innocuous forms, processes other than HDS have to be employed.

One of the most interesting options is oxidative desulfurization (ODS) [2]. The greatest advantage of ODS as compared with the conventional HDS processes is that it can be carried out in liquid phase under very mild conditions- at near room temperature and under atmospheric pressure. In ODS reactions, the divalent sulfur can be oxidized by the electrophilic addition reaction of oxygen atoms to the hexavalent sulfur of sulphones. The chemical and physical properties of sulphones are significantly different from those of hydrocarbons in fuel oil. Therefore, they can easily be removed by separation operations such as distillation, solvent extraction, adsorption, and decomposition.

Among these oxidants, $\mathrm{H}_{2} \mathrm{O}_{2}$ is mostly chosen as an oxidant, only producing water as a byproduct [3]-[15]. Peracids produced in situ from organic acids catalysts and $\mathrm{H}_{2} \mathrm{O}_{2}$ are reported to be very effective for rapid oxidation of sulfur compounds in fuel oils under mild conditions. Heteropolyacid catalysts in $\mathrm{H}_{2} \mathrm{O}_{2}$ oxidation system have also exhibited high catalytic activity for the oxidation of BTs and DBTs [7],[8]. We have shown that a phase transfer catalytic system can be very effective in the ODS reaction [4]. However, the main obstacles for these catalysts to the industrial application of the process at present are the difficulties in separation and recovery. Therefore, the use of solid catalysts in ODS processes has been developed in recent years. Many types of solid catalysts have been attempted, such as $\mathrm{Ti}$ molecular sieves [9]-[12], $\mathrm{WO}_{\mathrm{x}} / \mathrm{ZrO}_{2}$ [13],[14], vanadium oxide [15],[16] and molybdenum supported catalysts [17],[18]. However, the selectivity of these catalysts for sulfides in fuels is not high, and some components of the fuel are also oxidized. Therefore, the solid catalysts with high selectivity is highly desirable in heterogeneous ODS system. Some attempts have been done for the heterogeneization of tungsten acid compounds by immobilization on a silica support with the use of silica-bound alkyl ammonium salts [19],[20]. 
Within this context, the aim of the present study was two-fold. We have studied the oxidation with $\mathrm{H}_{2} \mathrm{O}_{2}$ of several model molecules in a Liquid-Liquid ( $\mathrm{L}-\mathrm{L}$ ) phase system in the presence of heterogeneous catalysts. The results reported here provide basic information about the applicability of the oxidation method with hydrogen peroxide in the selective transformation of organic sulfur compounds contained in kerosene and light oils.

\section{EXPERIMENTAL}

\section{Catalyst preparation}

Catalysts were prepared by ionic exchange of quaternary ammonium functionalized silica supports (Silicycle: SiliaBond ${ }^{\circledR}$ TMA Chloride). An excess (3:1) of metallic salts $\left(\mathrm{Na}_{2} \mathrm{MoO}_{4}, \mathrm{Na}_{2} \mathrm{WO}_{4}\right.$, phosphomolybdic acid and phosphotungstic acid) were solubilized in water (25 mL), and then $4 \mathrm{~g}$ of functionalized silica was added. The suspension was maintained under vigorous stirring at room temperature for $24 \mathrm{~h}$. The solid was filtered off and washed ten times with $25 \mathrm{~mL}$ of water and dried at room temperature. The codes of the samples are compiled in Table 1.

\section{Catalyst characterization}

The metal loading of the catalysts was determined using inductively coupled plasma absorption spectrometry, with a Perkin-Elmer Optima 3300 DV instrument. Ultraviolet-visible spectra were measured on a Varian Cary 5000 UV-vis spectrophotometer equipped with an integrating sphere. A $\mathrm{BaSO}_{4}$ disc was used as reference. All spectra were acquired under ambient conditions.

X-ray photoelectron spectra were recorded with a VG Escalab 200R spectrometer, equipped with a hemispherical electron analyzer and an $\mathrm{Mg} \mathrm{K \alpha}(\mathrm{hv}=1253.6 \mathrm{eV}) \mathrm{X}$-ray source. The binding energies (BE) were referenced to the $\mathrm{C}$ 1s peak at $284.9 \mathrm{eV}$. Atomic surface contents were estimated from the areas of the peaks, corrected using the corresponding sensitivity factors.

The liquid-phase catalytic sulfoxidation of sulfur-containing organic compounds with hydrogen peroxide was carried out batchwise in a mechanically stirred $250-\mathrm{mL}$ thermostated glass reactor equipped with thermometer, reflux condenser, and a septum for withdrawing samples. In a typical experiment, $90 \mathrm{~g}$ of the sulfur compound solution in n-hexadecane (Aldrich) were heated to the reaction temperature. Then, a solution of hydrogen peroxide (70 \% w/w, kindly supplied by Solvay Química, S.L.) mixed with the solvent was added to the apolar phase, and finally $0.25 \mathrm{~g}$ of catalyst was added to the reactor. Aliquots were taken from the reactor at different reaction times; the total 
amount withdrawn from the reactor was less than $10 \%$ in order to avoid interferences in the reaction results due to changes in the total mass inside the reactor. The apolar phase was recovered by decantation and analysed by GC-FID equipped with a capillary column (HP-WAX, 25 m, $\varnothing=$ $0.53 \mathrm{~mm}, 1.0 \mu \mathrm{m}$ film thickness). The hydrogen peroxide concentration was determined by standard iodometric titration.

\section{RESULTS AND DISCUSSION}

The amount of metal incorporated into the samples (Table 1) is very similar in all samples. This finding can be ascribed to the fact that the exchange procedure, which depends on the number of ammonium groups on the funzionalized silica surface, is similar in all samples.

The DRS-UV-Vis spectra of samples show absorption associated with the ligand metal charge transfer (LMCT) from the oxygen to the transition metal ion (Figure 1). Mo-TMA sample spectrum shows an absorption band at $256 \mathrm{~nm}$, which is characteristic of molybdate species (Mo(IV)) [21]. The electronic spectrum of MoP-TMA sample is somewhat more complex showing two maxima at 214 and $300 \mathrm{~nm}$ which is typical of ammonium phosphomolybdate salt [22]. Tungsten-containing samples are similar but the peaks shift to lower wavelengths, W-TMA spectrum shows a peak around $200 \mathrm{~nm}$, usually present in tungstic acid [23], but WP-TMA spectrum exhibits two peaks at 200 and $252 \mathrm{~nm}$, which are assigned to phosphotungstic acid [24].

The binding energies of the most intense $\mathrm{Mo3d}_{5 / 2}$ and $\mathrm{W}_{4} \mathrm{f}_{7 / 2}$ peaks indicate that the ions remain in the highest oxidation state $\left(\mathrm{Mo}^{6+} \mathrm{BE}=232.0 \mathrm{eV}\right.$ and $\mathrm{W}^{6+} \mathrm{BE}=36.0 \mathrm{eV}$, respectively). No change has been observed in the value of the binding energy irrespectively of the transition element and presence of phosphorus.

Summing up, the data provided by both UV-vis and photoelectron spectroscopic techniques confirm unambiguously that the oxidation state and anion structure of tungsten and molybdenum compounds remain unchanged after the ion exchange.

\section{Influence of the effect of the catalysts nature}

All the samples synthesized were then tested in the removal of S-containing compounds in a fuel by means of oxidative desulfurization (ODS) reaction. For the sake of ease understanding, a model synthetic fuel consisting of a solution of DBT in n-hexadecane was used. The oxidant employed consisted in a hydrogen peroxide solution in acetonitrile. The concentration of DBT was set at 0.2 
wt \% (240 ppm S). Although all catalysts were active in the ODS reaction (Figure 2) some differences were observed among the samples used. Molybdenum catalysts are clearly more active than tungsten counterparts (Figure 2). At short reaction time molybdenum catalysts reach high DBT conversion levels, while the reaction is slower for tungsten samples. In addition, the P-containing metal catalysts are more active than their P-free counterparts. This effect is evident for both molybdenum- and tungsten-containing catalysts (Figure 2). Based on these preliminary results, as the MoP-TMA catalyst prepared with phosphomolybdic acid displayed better performance in ODS reaction, it was selected for the next steps of the study, similar results have been found with homogeneous systems [25].

\section{Influence of the reaction temperature}

The effect of temperature on the kinetics of the elimination of DBT was investigated (333-353 K). The conversion profiles of dibenzothiophene in the ODS reaction versus the reaction time at three different temperatures (333, 343 and $353 \mathrm{~K}$ ) are shown in Figure 3. A rise in the reaction temperature from 333 to $343 \mathrm{~K}$ led to a remarkable increase in the reaction rate at every time of reaction. However, this increase in the temperature from 343 to $353 \mathrm{~K}$ was less marked. Moreover, the conversion of DBT was complete in only $30 \mathrm{~min}$ of reaction time at $353 \mathrm{~K}$, however at $333 \mathrm{~K}$ only $55 \%$ of the DBT conversion was reached. In view of these results, the reaction temperature employed for next studies was set at $353 \mathrm{~K}$. Though this temperature is the highest investigated, a very high excess of hydrogen peroxide was used in this study, which is quite far from an industrial application. Therefore it is inferred that reaction rate will decrease when hydrogen peroxide concentration is decreased [4].

\section{Influence of the $\mathrm{H}_{2} \mathrm{O}_{2}$ : substrate ratio}

The above experiments were carried out with an excess of hydrogen peroxide $\left(\mathrm{H}_{2} \mathrm{O}_{2} / \mathrm{DBT}=10\right.$ molar ratio). However, according to the stoichiometry only 2 mol of $\mathrm{H}_{2} \mathrm{O}_{2}$ are consumed per mole of sulphone ( $\left.\mathrm{R}-\mathrm{SO}_{2}\right)$ produced (see Scheme 1). Thus, it was of interest to explore the influence of the hydrogen peroxide concentration on the kinetics of DBT conversion. Several $\mathrm{H}_{2} \mathrm{O}_{2}$-to-substrate molar ratios were selected, including 10/1, 5/1 and 2.5/1. The lowest $\mathrm{H}_{2} \mathrm{O}_{2}$ concentration was set slightly above $\left(\mathrm{H}_{2} \mathrm{O}_{2} / \mathrm{DBT}=2.5 / 1\right.$ molar $)$ the stoichiometric $\left(\mathrm{H}_{2} \mathrm{O}_{2} / \mathrm{DBT}=2 / 1\right.$ molar $)$ ratio in order to avoid limitations of the oxidant reagent and to achieve complete conversion of DBT. The hydrogen peroxide-to-DBT ratio had a strong influence on the reaction rate (Figure 4). The reaction rate decreases with the $\mathrm{H}_{2} \mathrm{O}_{2}$ /DBT ratio. The reaction rate is the highest for $\mathrm{H}_{2} \mathrm{O}_{2} / \mathrm{DBT}=10 / 1$ molar ratio (Figure 4) for which complete DBT conversion was attained at $30 \mathrm{~min}$. However, although the 
reaction rate is lower with $\mathrm{H}_{2} \mathrm{O}_{2} / \mathrm{DBT}=2.5 / 1$ molar ratio, $100 \%$ of DBT conversion can be reached at the end of the reaction. Here, we show that complete DBT conversion is obtained with oxidantto-sulfur ratio close to the stoichiometry (2.5/1), and this ratio was used in the following studies, because this hydrogen peroxide is more interesting from the industrial point of view.

\section{Influence of the nature of the substrate}

DBT is representative of the S-compounds present in middle distillate fuels. However, other Scontaining molecules are present in liquid fuels, and their reactivity against ODS reaction should be evaluated. For this reason, we have compared the performance of our catalytic system in the ODS of other sulfur containing compounds: benzothiophene (BT), dibenzothiophene (DBT) and 4,6dimethyl-dibenzothiophene (DMDBT). The relative ODS rates of these three model S-containing molecules allow not only to rank them in terms of reactivity but also to evaluate the potential of the ODS process in fuel desulfurization. Comparison of the reactivity of different sulfur-containing compounds revealed that reactivity depends on the type of S-containing molecule to be oxidized. The relative ranking of the reaction rate was $\mathrm{DBT}>\mathrm{DMDBT} \approx \mathrm{BT}$ (Figure 5). In principle, this order of reactivity is unexpected because the BT molecule, with a relatively accessible S-atom, would give rise to a higher oxidation rate than in the more sterically hindered S-containing DBT and DMDBT molecule. The explanation for the above trend can be visualized in terms of S-C bond stabilization in the BT molecule. In the case of DBT, and to a lesser extent that of DMDBT, the inducing effect of the aromatic rings elicits an increase in the electron charge density of the S-atom, meaning that the reaction can be oxidized more easily by hydrogen peroxide.

\section{Solvent effects}

In all of previously depicted experiments, acetonitrile (ACN) was used as solvent. ACN is appropriate solvent because it is able to extract and solve the reaction products and exhibits a low surface tension, which facilitates the transfer of products and reagents at the polar-apolar interface, increasing notably the mass transfer along the interphase. However, ACN is partially dissolved in the apolar (fuel) phase [26], then ACN molecules are present in the fuel phase, in consequence the final nitrogen concentration in the fuel is very high. To avoid this, a later separation unit is mandatory to remove the fraction of ACN transferred to the fuel phase. Accordingly, other alternative solvents have also been explored in this work. Several solvents have been proposed in the bibliography but the best results in ODS has been reported using $\gamma$-butyrolactone (GBL) [27].

The nature of the solvent also plays a very important role in liquid-phase catalytic reactions. The solvent has an important effect on the outcome of the reaction, yields, by-product formation, and 
reaction kinetics, although this effect is strongly dependent on the type of catalyst used and on the nature of the substrate. The DBT conversion profile versus time (Figure 6) shows a lower activity in the experiment with GBL solvent, when standard reaction conditions are used (stirring: $400 \mathrm{rpm}$ ). GBL is quite more viscous than ACN and in consequence there are some mass transfer limitations at low stirring rate. But, the same experiment at high stirring speed (low mass transfer limitations) GBL yields to slightly higher conversion level than ACN (Figure 6).

\section{Catalyst reuse}

Finally, we carried out catalyst reuse experiments. The filtered catalyst was dried after washing with acetonitrile and reused without further treatment. Surprisingly, the catalytic activity increases with operation cycles, reaching a maximum after 5 operation cycles (Figure 7). The used catalysts after 5 operation cycles were analyzed by XPS. It was shown that the binding energy of the most intense Mo3d $_{5 / 2}$ peak $(B E=233.0 \mathrm{eV})$ shifts by about $1 \mathrm{eV}$ toward higher binding energy with respect to the fresh sample $(\mathrm{BE}=232.0 \mathrm{eV})$ (Figure 8). This change is attributed to the formation of peroxomolybdates moieties (Scheme 2). This molybdenum species are formed during the activation of polymolybdophosphates with hydrogen peroxide in organic solution [28]-[31], and they are very active in oxidation reactions [32]. Based on this finding, we decide to activate a fresh MoP-TMA fresh catalyst with a $\mathrm{H}_{2} \mathrm{O}_{2}$ solution in acetonitrile before their use in reaction. Sample was activated adding $1 \mathrm{~g}$ catalyst to $60 \mathrm{ml}\left(5 \mathrm{wt} \% \mathrm{H}_{2} \mathrm{O}_{2}\right.$ in $\mathrm{ACN}$ ) under stirring for $30 \mathrm{~min}$. This activated catalyst became highly active, and their catalytic activity is very close to that reused along 5 cycles. This activated catalyst was reused 4 times in reaction without activity drop (Figure 9). Taking in account these changes in the catalytic activity of MoP-TMA after activation with hydrogen peroxide, Mo-TMA catalysts was activated also and tested in reaction, this activated catalyst was more active than the fresh one, but it yields a lower activity than activated MoP-TMA, similar observation obtained with fresh samples.

\section{CONCLUSIONS}

This work demonstrates that the desulfurization of DBT, and also of BT and DMDBT, can be effectively accomplished by oxidation with hydrogen peroxide under very mild reaction conditions; namely atmospheric pressure and temperatures close to ambient (333-353 K). S removal by the ODS reaction from BT, DBT and DMDBT is easy and can be almost completed under the very mild conditions imposed by this reaction. This very high reactivity makes the ODS reaction promising for the deep desulfurization of middle distillates. 
The ODS process can be considered as no competitor of thee traditional HDS one. It appears to be complementary of it since hydrotreatments not only remove $\mathrm{S}$ and other heteroatoms from refinery streams but also improve the quality of the fuels. The ODS is specifically designed to be integrated in the last processing steps to decrease remaining sulphur until the levels fixed by environmental legislations. Thus, it seems appropriate to compare the ODS with the revamping or modification of an HDS unit able to perform a deep desulfurization and reach the same low S-levels which can be achieved with the coupled standard HDS and ODS technologies.

Molybdenum catalysts are modified by contact with hydrogen peroxide yielding peroxomolybdates moieties; these species are more active than acid precursors. Fresh molybdenum catalysts can be activated with hydrogen peroxide, and these activated systems are very active in ODS reaction and can be reused four times without lose in activity.

\section{References}

[1] C. Song, X. Ma, Appl. Catal. B: Environ. 41 (2003) 207.

[2] A. Anisimov, A. Tarakanova, Russ. J. Gen. Chem. 79 (2009) 1264.

[3] S. Otsuki, T. Nonaka, N. Takashima, W.H. Qian, A. Ishihara, T. Imai and T. Kabe, Energy Fuels 14 (2000) 1232.

[4] J.M. Campos-Martin, M.C. Capel-Sanchez, J.L.G. Fierro, Green Chem. 6 (2004) 557.

[5] Y. Shiriraishi, T. Naito, T. Hirai, Ind. Eng. Chem. Res. 42(24) (2003) 6034.

[6] L.Y. Kong, G. Li, X.S. Wang, B. Wu, Energy Fuels 20 (2006) 896.

[7] F.M. Collins, A.R. Lucy, C. Sharp, J. Mol. Catal. A: Chem. 117 (1997) 397.

[8] H. Mei, B.W. Mei, T.F. Yen, Fuel 82 (2003) 405.

[9] V. Hulea, F. Fajula, J. Bousquets, J. Catal. 198 (2001) 179.

[10] Y. Shiraishi, T. Hirai, I. Komasawa, J. Chem. Eng. Jpn. 35 (2002) 1305.

[11] L.Y. Kong, G. Li, X. S.Wang, Catal. Today 93-95 (2004) 341.

[12] S.F. Cheng, Y.M. Liu, J.B. Gao, L.L. Wang, X.L. Liu, G.H. Gao, P. Wu, M.Y. He, Chin. J. Catal. 27 (2006) 547

[13] E. Torres-Garcia, G. Canizal, S. Velumani, L.F. Ramirez-Verduzco, F. Murrieta-Guevara, J.A. Ascencio, Appl. Phys. A 79 (2004) 2037.

[14] X.-M. Yan, P. Mei, J. Lei, Y. Mi, L. Xiong and L. Guo, J. Mol. Catal. A: Chem. 304 (2009) 52.

[15] Y. Shiraishi, T. Naito, T. Hirai, Ind. Eng. Chem. Res. 42 (2003) 6034.

[16] H. Gómez-Bernal, L. Cedeño-Caero, A. Gutiérrez-Alejandre, Catal. Today 142 (2009) 227.

[17] J.L. Garcia-Gutierrez, G.A. Fuentes, M.E. Hernandez-Teran, F. Murrieta, J. Navarrete, F. Jimenez-Cruz, Appl. Catal. A-Gen. 305 (2006) 15.

[18] V.V.D.N. Prasad, K.-E. Jeong, H.-J. Chae, C.-U. Kim, S.-Y. Jeong, Catal. Commun. 9 (2008) 1966.

[19] F. Bigi, A. Corradini, C. Quarantelli, G. Sartori, J. Catal. 250 (2007) 222.

[20] B. Sreedhar, P. Radhika, B. Neelima, N. Hebalkar and A.K. Mishra, Catal. Commun. 10 (2008) 39.

[21] A. Köckritza, M. Kanta, M. Walterb, A. Martin, Appl. Catal. A: Gen. 334(1-2) (2008) 112.

[22] G. Ranga Rao, T. Rajkumara, B. Varghese, Solid State Sci. 11(1) (2009) 36.

[23] L. Barrio, J.M. Campos-Martin, J.L.G. Fierro, J. Phys. Chem. A 111 (2007) 2166.

[24] A. Kukovecz, Z. Kónya, I. Kiricsi, J. Mol. Struct. 563-564 (2001) 409. 
[25] A. De Angelis, P. Pollesel, D. Molinari, W.O. Parker Jr, A. Frattini, F. Cavani, S. Martins and C. Perego, Pure Appl. Chem., 79 (2007) 1887.

[26] V.P. Sazonov, D.G. Shaw, J. Phys. Chem. Ref. Data 31(4) (2002) 989.

[27] L.F. Ramirez-Verduzco, E. Torres-Garcia, R. Gomez-Quintana, V. Gonzalez-Pena and F. Murrieta-Guevara, Catal. Today 98 (2004) 289.

[28] L. Salles, R. Thouvenot and J.-M. Brégeault, Dalton Trans. (2004) 904.

[29] L. Salles, C. Aubry, R. Thouvenot, F. Robert, C. Dorémieux-Morin, G. Chottard, H. Ledon, Y. Jeannin and J.-M. Brégeault, Inorg. Chem. 33 (1994) 871

[30] L. Salles, C. Aubry, F. Robert, G. Chottard, R. Thouvenot, H. Ledon and J.-M. Brégeault, New J. Chem. 17 (1993) 367

[31] C. Aubry, G. Chottard, N. Platzer, J.-M. Brégeault, R. Thouvenot, F. Chauveau, C. Huet and H. Ledon, Inorg. Chem. 30 (1991) 4409

[32] G. Wahl, D. Kleinhenz, A. Schorm, J. Sundermeyer, R. Stowasser, C. Rummey, G. Bringmann, C. Fickert, W. Kiefer, Chem. Eur. J. 5(11) (1999) 3237. 
Table 1 Samples prepared and metal content determined by chemical analysis

\begin{tabular}{|l|l|c|}
\hline Sample & Metal precursor & $\begin{array}{c}\text { Metal content } \\
\text { (wt \%) }\end{array}$ \\
\hline Mo-TMA & $\mathrm{H}_{2} \mathrm{MoO}_{4}$ & 11.3 \\
\hline MoP-TMA & $\mathrm{H}_{3} \mathrm{PMo}_{12} \mathrm{O}_{40}$ & 12.2 \\
\hline W-TMA & $\mathrm{H}_{2} \mathrm{WO}_{4}$ & 12.4 \\
\hline WP-TMA & $\mathrm{H}_{3} \mathrm{PW}_{12} \mathrm{O}_{40}$ & 12.4 \\
\hline
\end{tabular}




\section{Figure Captions}

Scheme 1 Simplified reactions of sulfur compounds following the ODS process

Scheme 2 Schematic formation of peroxomolybdates

Figure 1 UV-Vis spectra of prepared samples

Figure 2 Influence of the catalyst nature in the conversion of dibenzothiophene. Conditions: Substrate: $\mathrm{H}_{2} \mathrm{O}_{2}=1: 10$ (molar ratio), T: $353 \mathrm{~K}$, solvent: Acetonitrile, $0.2 \mathrm{wt} \%$ DBT in hexadecane.

Figure 3 Influence of the reaction temperature in the conversion of dibenzothiophene. Conditions: Substrate: $\mathrm{H}_{2} \mathrm{O}_{2}=1: 10$ (molar ratio), solvent: Acetonitrile, $0.2 \mathrm{wt} \% \mathrm{DBT}$ in hexadecane, 0.25 g catalyst MoP-TMA.

Figure 4 Influence of the $\mathrm{H}_{2} \mathrm{O}_{2}$ :substrate ratio in the conversion of dibenzothiophene. Conditions: Solvent: Acetonitrile, 0.2 wt \% DBT in hexadecane, 0.25 g catalyst MoP-TMA, T: 353 K.

Figure 5 Conversion-time curves for different S-compounds. Conditions: Solvent: Acetonitrile, $0.2 \mathrm{wt} \%$ of S-compound in hexadecane, $0.25 \mathrm{~g}$ catalyst MoP-TMA, T: $353 \mathrm{~K}$.

Figure 6 Influence of solvent in the conversion of dibenzothiophene. Conditions: $0.2 \mathrm{wt} \% \mathrm{DBT}$ in hexadecane (240 ppm S), substrate: $\mathrm{H}_{2} \mathrm{O}_{2}=2.5: 1$ (molar ratio), $\mathrm{T}=353 \mathrm{~K}$.

Figure 7 Influence of the reuse of catalyst in the conversion of dibenzothiophene. Conditions: 0.2 wt\% DBT in hexadecane (240 ppm S), substrate: $\mathrm{H}_{2} \mathrm{O}_{2}:=2.5: 1$ (molar ratio), $\mathrm{T}=353 \mathrm{~K}$.

Figure 8 Mo3d core-level spectra of fresh, reused and activated MoP-TMA sample.

Figure 9 DBT conversion in several reaction cycles of the activated catalyst at $15 \mathrm{~min}$. Conditions: $0.2 \mathrm{wt} \%$ DBT in hexadecane (240 ppm S), substrate: $\mathrm{H}_{2} \mathrm{O}_{2}:=2.5: 1$ (molar ratio), $\mathrm{T}=353 \mathrm{~K}$ 


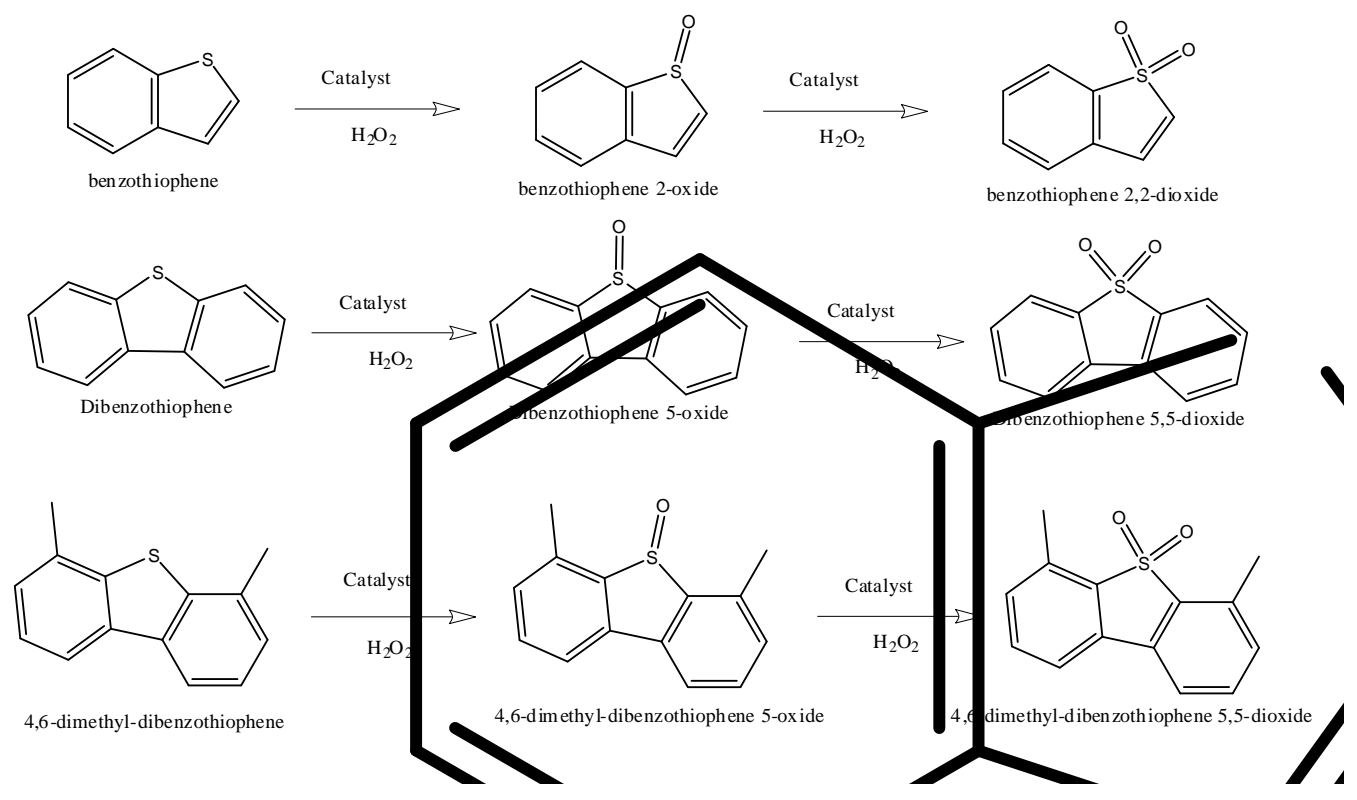

Scheme 1

Scheme 2 

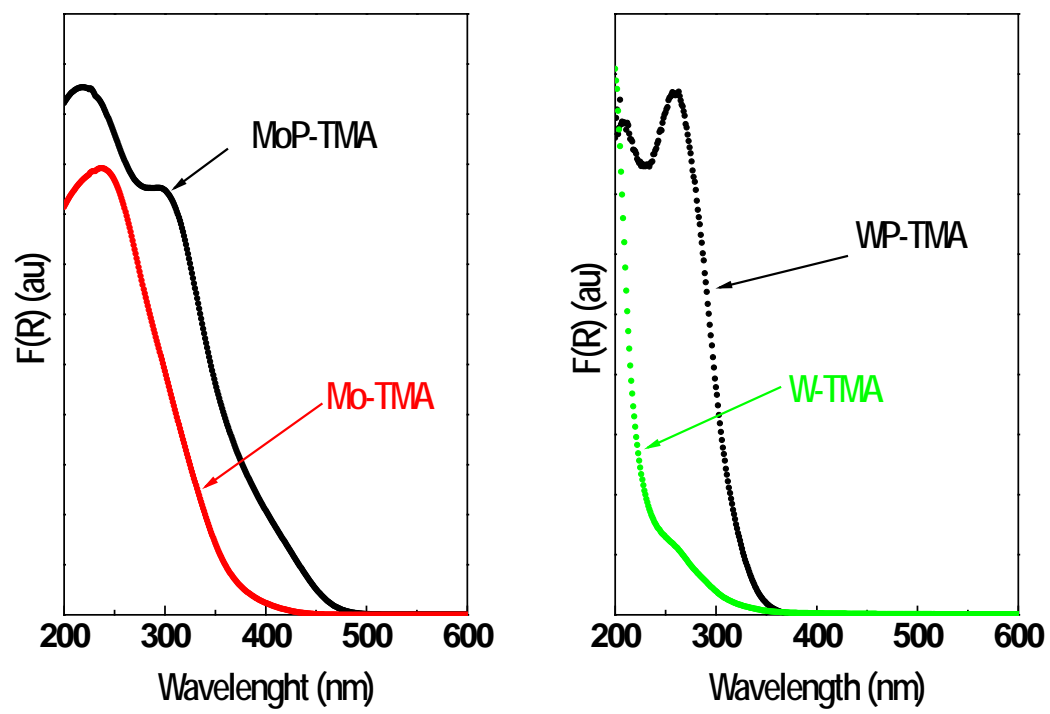

Figure 1

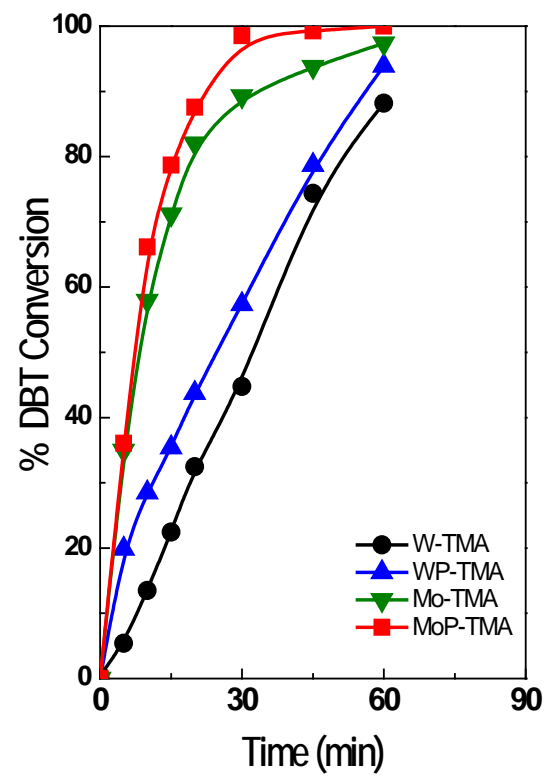

Figure 2 


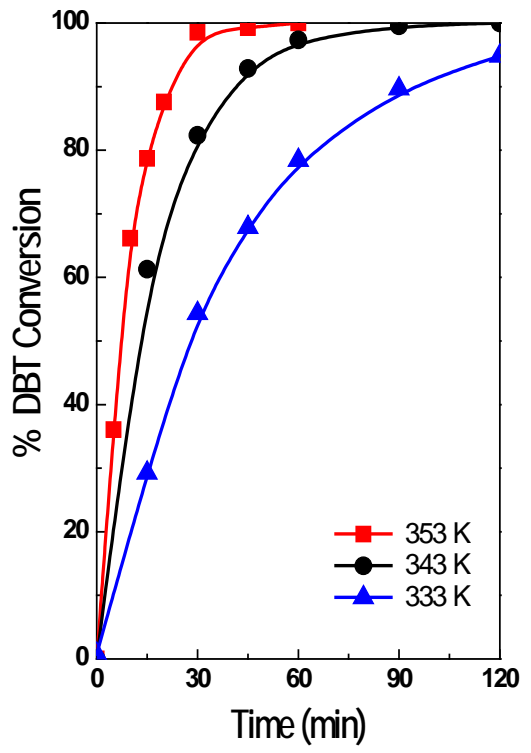

Figure 3

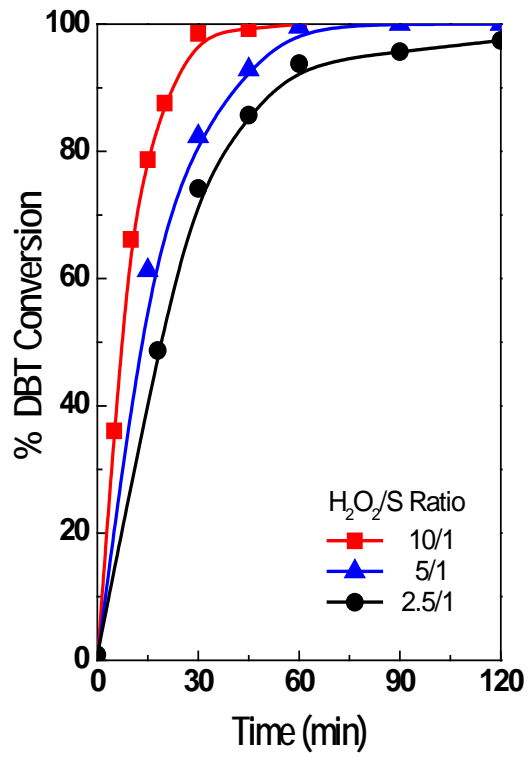

Figure 4 


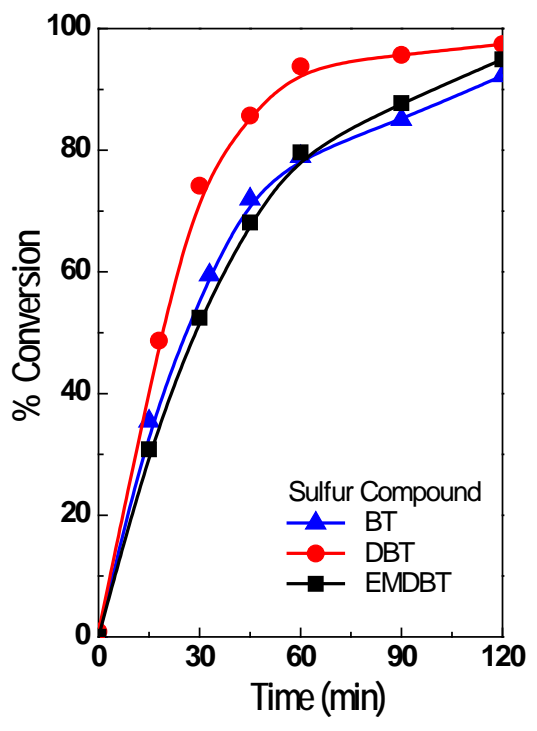

Figure 5
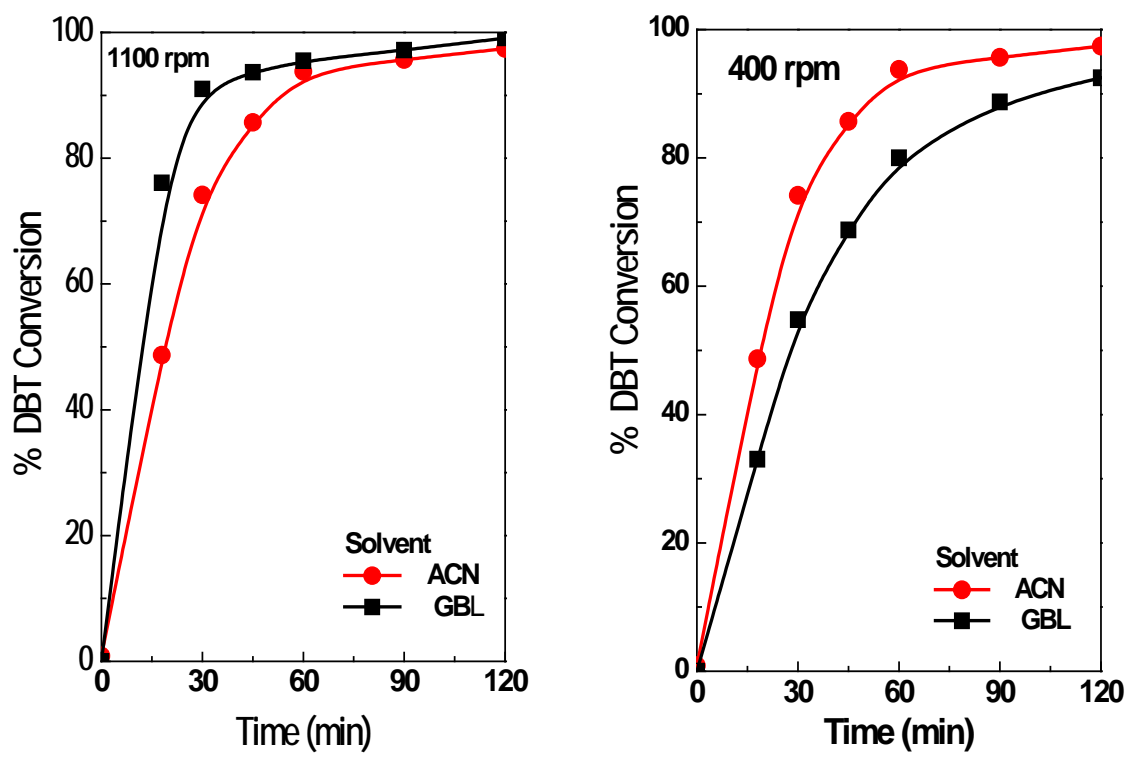

Figure 6 


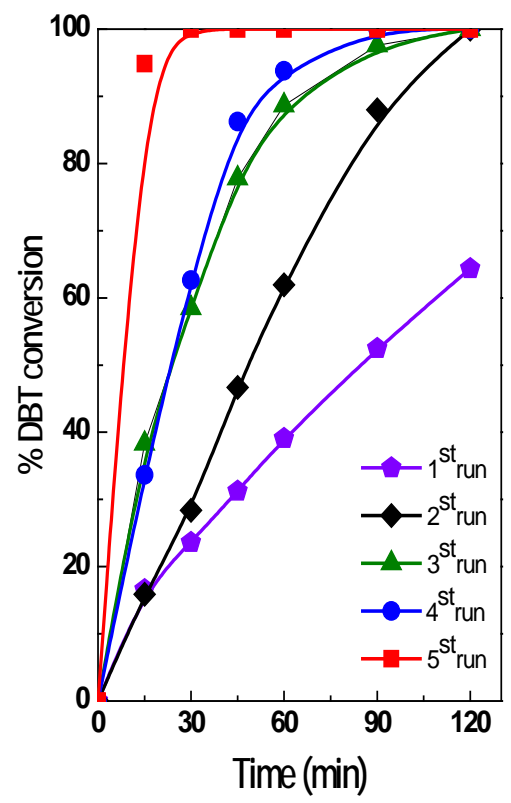

Figure 7
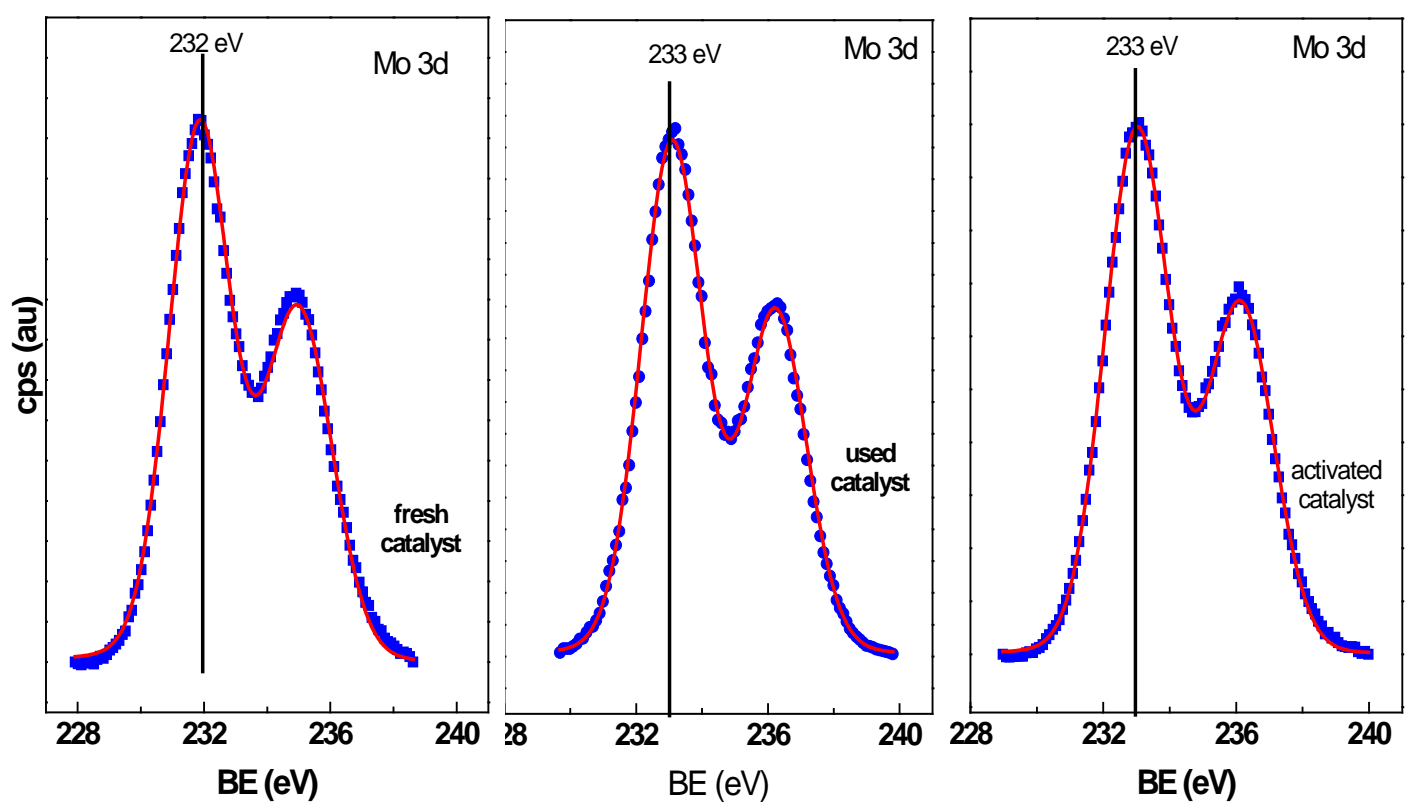

Figure 8 


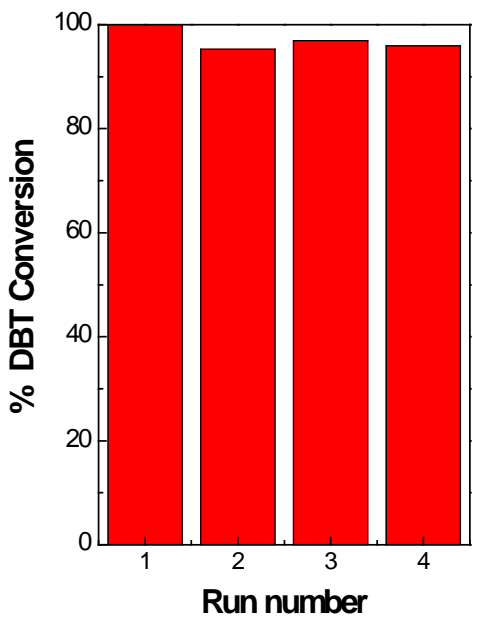

Figure 9 